\title{
Mediation and Modification of the Association Between Hopelessness, Hostility, and Progression of Carotid Atherosclerosis
}

\author{
Ricardo A. Pollitt, ${ }^{1,6}$ Mark Daniel, ${ }^{1,2}$ Jay S. Kaufman, ${ }^{1}$ John W. Lynch, ${ }^{3}$ \\ Jukka T. Salonen, ${ }^{4,5}$ and George A. Kaplan ${ }^{3}$
}

Accepted for publication: March 29, 2004

Hopelessness and hostility are linked to progression of carotid atherosclerosis (PCA). The purpose of this study was to replicate such relations and to evaluate the role of biological pathways involving hyperactivation of the sympathetic nervous system (SNS) and the hypothalamic-pituitary-adrenocortical (HPA) axis. PCA was evaluated by 4-year change in three ultrasound measures of intima-media thickness (IMT) in 1027 men aged 42-60 years at baseline. Effect modification and mediation of relationships between psychosocial factors and PCA were examined for the measures systolic blood pressure (SBP), fibrinogen, and waist-to-hip ratio (WHR), levels of which are indicative of activity along these pathways. Hopelessness and hostility were associated with PCA. Fibrinogen mediated to a moderate extent the association between hopelessness and PCA. SBP significantly modified the relation between hostility and PCA in participants of moderate hostility. The above biological pathways are implicated in the mechanisms connecting hopelessness, hostility, and PCA.

KEY WORDS: hopelessness; hostility; IMT; SBP; fibrinogen.

\section{INTRODUCTION}

Psychological conditions such as depression, hopelessness, and hostility are associated with an elevated risk of cardiovascular disease and allcause mortality. Depression has been associated with

\footnotetext{
${ }^{1}$ Department of Epidemiology, The University of North Carolina at Chapel Hill, Chapel Hill, North Carolina.

${ }^{2}$ Département de Médecine Sociale et Preventive, l' Université de Montréal, Montreal, Quebec.

${ }^{3}$ Department of Epidemiology, Center for Social Epidemiology and Population Health, The University of Michigan, Ann Arbor, Michigan.

${ }^{4}$ Research Institute of Public Health and Department of Public Health and General Practice, The University of Kuopio, Kuopio, Finland.

${ }^{5}$ Inner Savo Health Centre, Suonenjoki, Finland.

${ }^{6}$ To whom Correspondence should be addressed at Department of Epidemiology, School of Public Health, The University of North Carolina, Campus Box \# 7435, Chapel Hill, North Carolina 27599-7435; e-mail: pollitt@email.unc.edu.
}

an increased risk of developing and dying from cardiovascular disease (CVD) (Anda et al., 1993; Aromaa et al., 1994; Bruce et al., 1994; Everson et al., 1996, 1997a; Glassman and Shapiro, 1998; Musselman et al., 1998; Rozanski et al., 1999; Rugulies, 2002). A related psychological construct, hopelessness, is implicated as conferring an independent risk of developing CVD. While lack of hope is often considered an integral part of depression (Brown and Harris, 1978), hopelessness may function independently from depression (Alloy et al., 1988; Greene, 1989). Observational studies and animal models of hopelessness have linked hopelessness with an elevated risk of sudden death and CVD (Anda et al., 1993; Bruhn et al., 1974; Engel, 1968; Ritcher, 1957). The Kuopio Ischemic Heart Disease (KIHD) study cohort showed that middleaged men with high levels of hopelessness had a greater risk of incident myocardial infarction (MI) and CVD-specific and all-cause mortality (Everson et al., 1996, 1997a). Such men also developed more 
atherosclerosis over a 4-year interval, independent of depression and traditional cardiovascular risk factors.

Hostility is associated with negative affective characteristics including suspicion, cynicism, anger, aggression, and irritability (Julkunen et al., 1994; Maruta et al., 1993). Analyses of the KIHD study showed that men with high cynical distrust and anger-control scores had close to twice the level of progression of carotid atherosclerosis over a 2-year period than men with lower scores (Julkunen et al., 1994). Adjustments for various biological and socioeconomic risk factors eliminated these associations, however. Similarly, men with hostility scores in the top quartile had more than twice the risk of all-cause mortality, cardiovascular mortality, and $\mathrm{MI}$ as compared to men with scores in the lowest quartile (Everson et al., 1997b). In this study, SES and biological risk factors only slightly attenuated the associations, whereas behavioral risk factors considerably attenuated them. Some, but not all, studies of these relationships in other populations have reported an association between hostility and an elevated risk of CVD (Barefoot et al., 1995; Hearn et al., 1989; Iribarren et al., 2000; Maruta et al., 1993; Matthews et al., 1998).

Unhealthful behavior is known to mediate, in part, associations between negative psychological conditions and CVD risk (Glassman et al., 1990; Kaplan and Keil, 1993; Musselman et al., 1998; Niedhammer et al., 1998; Rozanski et al., 1999; Twisk et al., 2001; Ziegelstein et al., 1998). Recent studies have also implicated neuroendocrine pathways in the mechanisms underlying the link between psychosocial state and risk of CVD (Anda et al., 1993; Everson et al., 1997b; Markowe et al., 1985; McEwen and Stellar, 1993; Miller et al., 2002; Pope and Smith, 1991; Suarez et al., 1998). Persons with depressive disorders may experience chronic hyperactivation of the sympathetic nervous system (SNS), and of the sympathetic-adrenal medullary (SAM) system specifically, resulting in greater blood pressure and heart rate, constriction of peripheral blood vessels, and higher circulating levels of epinephrine and norepinephrine (Cohen and Rodriguez, 1995; LaghrissiThode et al., 1997; Musselman et al., 1996). High hostility individuals also experience SNS hyperactivity (Rozanski et al., 1999; Suarez and Blumenthal, 1991; Suarez et al., 1998).

Depressed persons can suffer chronic stimulation of the hypothalamic-pituitary-adrenocortical (HPA) axis, with consequent elevated secretion of glucocorticoids, leading to increased release of insulin and hyperlipidemia, hypercholesterolemia, hypertension, hypertrophy of the adrenal gland, and injury to the intima and vascular endothelial cells (Bjorntorp, 1997; Checkley, 1996; Cohen and Rodriguez, 1995; Tsigos and Chrousos, 2002). Depression may increase the risk of cardiac events by way of reduced heart rate variability and impaired vagal control (Musselman et al., 1998; Rozanski et al., 1999). Hostility is also associated with a heightened reactivity of the HPA axis, high platelet reactivity, high daily-life ambulatory blood pressure, hypercortisolemia, and elevated circulating levels of catecholamines, along with a clustering of unhealthy lifestyle behaviors (Everson et al., 1997b; Pope and Smith, 1991; Rozanski et al., 1999; Suarez et al., 1998).

A greater understanding is needed of the biological pathways linking affective disorders and CVD. One way in which the influence of these pathways can be examined is by determining the extent to which levels of physiological measures linked to overactivity of the SNS or the HPA axis mediate or moderate some or all of the association between affective disorders and CVD. Negative affective states such as hopelessness or hostility likely influence human biology over both the shortand the long-term (Kelly et al., 1997; McEwen, 1998). Intermediate biological changes along the SNS or HPA axes, detectable by changes in physiological markers, may be involved in mediating longer-term health outcomes. On the other hand, it may be the case that specific physiologic measures involved in these biological pathways simply covary with the states of hopelessness or hostility, serving perhaps in the short term to modify, rather than mediate, the effects of negative affective states on the development of atherosclerosis over 4 years.

In an attempt to further describe the impact of activity along these pathways, the present study sought to examine the influence of three physiological measures linked to SNS and/or HPA activity in the association between affective disorders and CVD previously observed in the KIHD Study. Specifically, we evaluated the exploratory hypotheses that the influences of hopelessness and hostility on 4-year progression of carotid atherosclerosis (PCA) are modified or mediated by plasma fibrinogen, systolic blood pressure (SBP), and waist-to-hip ratio (WHR). Each of these variables has been previously implicated in the pathways by which affective disorders are linked to CVD (Bjorntorp, 1997; Glassman et al., 1990; Glassman and Shapiro, 1998; Musselman et al., 1996; Pratt et al., 1996; Rozanski et al., 1999). Plasma fibrinogen is an acute phase reactant and marker of 
inflammation in the cardiovascular system. It plays a fundamental role in thrombus formation, strongly affects platelet aggregation, and its concentration increases in response to a wide array of stressors in humans (Kelly et al., 1997; Saadeddin et al., 2002). Fibrinogen is also independently associated with depression and the related condition of vital exhaustion (Kop, 1999; Kop et al., 2002). Hypertension, an important independent risk factor for CHD, has been shown to develop in response to the administration of corticosteroids, and may result from hyperactivity of the SAM axis, which can occur in depressed and high-hostility individuals (Bjorntorp, 1997; Musselman et al., 1998). Abdominal adipose tissue indexed by WHR is linked to insulin resistance, and is a stronger risk factor than obesity for CHD, stroke, and mortality (Ferland et al., 1989). Insulin resistance and its sequelae may reflect hyperactivity of the HPA axis due to psychosocial stress (Bjorntorp, 1997; Kelly et al., 1997). Additionally, visceral obesity may be a function of elevated cortisol due to upregulated HPA activity (Mayo-Smith et al., 1989). Plasma fibrinogen, SBP, and WHR are thus each related to CVD and also potentially influenced by the pathophysiological responses that can occur in individuals with high hopelessness or hostility.

Analysis of mediation or effect modification by these measures has the potential to clarify the simple previously observed associations, and may thus lead to more sophisticated hypotheses to test whether (over)activity of the SNS and/or HPA axis is implicated in the link between negative affective states and CVD. Evidence for a biologically plausible mechanism of mediation would support a role for the SNS or HPA axis as a temporal intermediary between negative affective exposure and CVD risk. Evidence of effect modification by these biological measures could highlight the possible effects of a short-term biological response to a negative affective state on the long-term potential impact of such states on atherogenesis. Differentiation of the effects of the physiological measures is one of the steps needed in the process of unraveling the still poorly understood specific risks of negative affective states such as hopelessness or hostility.

\section{METHODS}

\section{Study Population}

The KIHD study was designed to investigate previously undetermined risk factors for ischemic heart disease, carotid atherosclerosis, and related outcomes in a population-based sample of middleaged men in eastern Finland, an area of high coronary morbidity and mortality (Keys, 1980; Salonen, 1988). Reports from the KIHD study have focused on the association between hostility and hopelessness, and PCA and cardiovascular mortality (Everson et al. 1996, 1997a,b; Greenglass and Julkunen, 1991; Julkunen et al., 1994; Lynch et al., 1998; Wilson et al., 1993). The study population and manner of recruitment have been described previously (Salonen, 1988). Briefly, in its entirety the KIHD study surveyed 2682 men $(82.9 \%$ of those eligible) aged 42, 48, 54, or 60 years at time of entry between March 1984 and December 1989. A followup evaluation, restricted to those who underwent ultrasound examination of the carotid arteries at baseline, was conducted on 1038 men. Fifty-two of the 1229 participants eligible for the follow-up evaluation could not participate on the basis of having moved, or because they were severely ill, or had died; a further 139 men refused to participate or could not be located. Data were obtained on 1027 men; there were 236, 242, 283, and 266 men in the 42-, 48-, 54-, and 60-year-old age groups, respectively. The mean follow-up duration was 4.2 years after the baseline survey (range: 3.9-5.1 years). For each participant, the follow-up survey was conducted during the same month, and at the same time of day, as the baseline survey conducted previously. For the purposes of the present analysis, complete covariate measures were available for 921 men in the analysis using hostility scores and 990 men in the analysis using hopelessness scores.

\section{Psychosocial Measures}

Hopelessness was assessed at baseline with two questionnaire items scored on a 5-point Likert scale (Everson et al., 1997a). These items were as follows: "I feel that it is impossible to reach the goals I would like to strive for" and "The future seems to me to be hopeless, and I can't believe that things are changing for the better." Questions were summed and the score distribution, ranging from 0 to 8 , divided into three levels: "Low hopelessness" (scores of 0,1 , or 2 , including $58.3 \%$ of all scores), "moderate hopelessness" (scores of 3, 4, or 5, representing $32.9 \%$ of the sample), and "high hopelessness" (scores of 6, 7, or 8, accounting for $8.8 \%$ of the sample) (Everson et al., 1997a). The "low" and "moderate" groups were pooled into a single category 
to enable greater statistical power. These categorizations were employed in order to further explain the specific relationships between psychosocial conditions and atherosclerosis previously reported (Everson et al., 1997a).

Hostility was assessed at baseline by selfreport using a questionnaire mailed to participants approximately half way through the first (2-year) follow-up period (Julkunen et al., 1994). This analysis restricts its consideration of hostility to cynical distrust (Greenglass and Julkunen, 1991), a measure of the cognitive component of hostility from the CookMedley Hostility Scale (Cook and Medley, 1954). Eight items from the cynical distrust scale were used. This abbreviated scale correlates with the original Cook-Medley scale $(r=0.77, p<0.001)$ and is held to be a valid, reliable, and specific measure of hostility and distrust. Cronbach's alpha for the Cynical Distrust Scale is 0.81, evidence of sufficient internal consistency (Everson et al., 1997b). Sample items from this scale are as follows: "It is safer to trust nobody" and "Most people make friends because friends are likely to be useful to them." Response options were on a 4-point Likert scale as follows: $0=$ completely agree $; 1=$ somewhat agree $; 2=$ somewhat disagree; 3 = completely disagree. Items were reverse scored and summed, providing a Cynical Distrust Scale score of from 0 to 24. Participants were grouped into Low, Moderate, and High hostility categories by tertiles. Tertile categorization was used instead of dichotomization (Julkunen et al., 1994) in order to further differentiate between subjective levels of hostility.

Marital status was categorized as either "single," "married," or "divorced/widowed." Smoking was assessed by questionnaire and persons classified as either "current smoker," "former smoker," or "never smoked." Treatment for hypertension or hyperlipidemia was assessed by medication usage. Education was recorded as number of years of total education completed. Season of the year was not included as a covariate given no evidence of confounding effects in previous KIHD analyses (Wilson et al., 1993). This present analysis used baseline values of psychosocial variables to predict 4-year change in intima-media thickness (IMT) measures.

\section{Biological Measures}

Biological measures included in this analysis are known to be associated with CVD-related mortality and acute myocardial infarction (Lynch et al., 1996).
Measures included plasma fibrinogen, WHR, and SBP. Fibrinogen was determined based on clotting of diluted plasma with excess thrombin using fresh samples after 12-h abstinence from food and coffee, and 3 -day abstinence from alcohol (Clauss, 1957). The coefficient of variation for the day-to-day measurement of variability for serum fibrinogen assays was $5.5 \%$. WHR was calculated as hip girth $(\mathrm{cm})$ into waist girth $(\mathrm{cm})$. BMI was not included in analyses given collinear relations with WHR. Three measures of SBP were taken with the participant supine, and averaged. This analysis used baseline values of biological measures in assessing interaction with baseline psychosocial measures to predict 4-year change in IMT. Treatment for hypertension was accounted for as a covariate in analyses, assessed by review of medications (Lynch et al., 1998).

\section{Carotid Atherosclerosis}

Carotid atherosclerosis was examined using high-resolution B-mode ultrasound measurements of intima-media thickness of a $1.0-1.5 \mathrm{~cm}$ section at the distal end of both the right and left common carotid artery (CCA) proximal to the carotid. Carotid atherosclerosis evaluated by B-mode ultrasound is an accepted measure of atherosclerosis and is associated with coronary artery disease (CAD) (Crouse, 2001; Crouse et al., 1996). The protocol for this procedure has been reported earlier (Salonen and Salonen, 1993). The precision of the measure is $\pm 0.03 \mathrm{~mm}$ (Salonen et al., 1993). Ultrasound images were focused on the posterior wall, with the subject supine, after a 15-min rest. IMT was assessed using Prosound software, which utilizes an edgedetection algorithm designed for ultrasound scanning enabling detection, tracking, and recording of the lumen/adventitia and media/adventitia interfaces (Selzer et al., 1994).

The present analysis used three measures of IMT, each assessed at baseline and at follow-up 4 years later. Mean IMT was defined as the mean of some 100 IMT readings taken from each CCA. Maximum IMT represents the average of the maximum IMT measured in the right and left CCA's. Plaque height was defined as the difference between the maximum and minimum IMT averaged over the left and right CCA. Each IMT measure represents a potentially different aspect of atherosclerotic progression, with changes in mean IMT serving as an overall measure of atherosclerotic progression 
(Lynch et al., 1997). Maximum IMT indicates the depth of the greatest lesion intruding into the lumen, while plaque height indicates the roughness of the arterial wall represented by the range of IMT and hence assessing how steeply atherosclerotic lesions protruded into the lumen. Progression of carotid atherosclerosis (PCA) was evaluated as the 4-year change in IMT value, recorded as the follow-up measure of IMT minus the baseline measure of IMT for each of the three IMT variables. These measures have been described more fully elsewhere (Lynch et al., 1997; Salonen, 1988).

\section{Data Analysis and Statistics}

We used linear regression models to examine relations between psychosocial measures of hopelessness and cynical hostility, and progression of atherosclerosis over 4 years (as estimated by mean change in three measures of IMT). The first set of models controlled for age and baseline level of carotid atherosclerosis; the second set of models also controlled for the covariates plasma fibrinogen, SBP, WHR, education level, smoking status, marital status, and treatment for hypertension. Slightly different numbers were used in these models compared to the analysis by Everson et al. due to the different covariates and categorizations used (Everson et al., 1997a).

Operationalization of the hostility and hopelessness exposure measures as indicator variables allowed for a thorough analysis of effect modification and mediation at different levels of exposure. Categorization of the exposures also obviated the need to transform these measures, as such transformation would have resulted in greater difficulty interpreting the results of analysis. Path analysis was considered, but the use of indicator variables made meeting this analytic technique's assumptions (e.g., linear relationship among the variables, interval scale data for all variables, uncorrelated residual error terms for endogenous variables) problematic (Bollen, 1998).

PCA was regressed (in separate models) on each psychosocial predictor. The determination of whether biological variables of SBP, WHR, and fibrinogen modified the effect of psychological predictors on PCA was made based on whether the addition of an interaction term representing a biological variable and the psychosocial predictor resulted in a significant $(p<0.05)$ change in the predictive capacity of the model, assessed by single and multiple partial $F$ tests (Kleinbaum et al., 1998).
The three biological variables were further examined as potential mediators of the relationship between the two psychosocial predictors and PCA. Mediation was defined in terms of the extent to which an intermediate variable "explains" an association between a predictor and a criterion, where the predictor is an independent variable, and the criterion is the dependent variable. A covariate may be a mediator according to the following conditions: 1 ) when the mediator is regressed on the exposure, the exposure must affect the mediator;2) when the outcome is regressed on the exposure, the exposure must affect the outcome; and 3) when the outcome is regressed on both the mediator and the exposure, the mediator must independently affect the outcome (Baron and Kenny, 1986; Hoyle and Kenny, 1999; Kenny et al., 1998).

To assess the potential mediating role of the biological variables plasma fibrinogen, SBP, and WHR, PCA was regressed (in separate models) on each of the psychosocial predictors with covariates included in the model to control for confounding (Hoyle and Kenny, 1999). The percentage change in the size of the beta coefficient for the psychosocial predictor when a potential biological mediator was removed from the model was calculated. A change of greater than $15 \%$ in the beta coefficient for the predictor was taken to suggest the presence of mediation; a change of greater than $30 \%$ was considered to strongly suggest mediation.

Analyses were conducted with the GLM and REG procedures in SAS version 8.2. Age-adjusted mean values of 4-year IMT were estimated and contrasted for each level of hopelessness and hostility while simultaneously controlling for confounders.

\section{RESULTS}

Descriptive statistics for the psychosocial predictor variables and covariates examined are given in Table I. There were 921 men with hostility data and complete IMT data; there were 990 men with hopelessness data and complete IMT data. Tables II and III present estimated mean 4-year change in IMT for each level of the three independent variables for these men. The cell sizes are smaller in Table III because complete covariate data were not available for all men. There were no significant differences in exposures or descriptive variables between the entire cohort and the subgroups for which complete covariates were 
Table I. Characteristics at Baseline for 1027 Eastern Finnish Men With Follow-up Measures for 4-Year Change in Intima-Media Thickness (IMT) ${ }^{a}$

\begin{tabular}{|c|c|c|}
\hline Variable & $n$ & Mean \pm SD \\
\hline Hostility score $^{b}$ & 921 & $12.57 \pm 4.19$ \\
\hline Hopelessness score ${ }^{c}$ & 990 & $2.48 \pm 1.93$ \\
\hline Waist-to-hip ratio (unitless) & 1025 & $0.94 \pm 0.06$ \\
\hline Age (years) & 1027 & $51.38 \pm 6.64$ \\
\hline Systolic blood pressure $(\mathrm{mmHg})$ & 1026 & $131.8 \pm 16.1$ \\
\hline Plasma fibrinogen $(\mathrm{g} / \mathrm{l})$ & 974 & $3.00 \pm 0.57$ \\
\hline \multirow[t]{2}{*}{ Education (years completed) } & 1024 & $9.2 \pm 3.5$ \\
\hline & $\%$ & \\
\hline \multicolumn{3}{|l|}{ Cigarette smoking $(n=1027)$} \\
\hline Never smokers & 273 & $26.6 \pm 1.4$ \\
\hline Former smokers & 406 & $39.5 \pm 1.5$ \\
\hline Current smokers & 348 & $33.9 \pm 1.5$ \\
\hline \multicolumn{3}{|l|}{ Marital status $(n=1025)$} \\
\hline Married/living as couple & 904 & $88.2 \pm 1.0$ \\
\hline Single & 57 & $5.6 \pm 0.7$ \\
\hline Divorced/widowed & 64 & $6.2 \pm 0.8$ \\
\hline \multicolumn{3}{|l|}{ Taking Hypertensive } \\
\hline \multicolumn{3}{|l|}{ Medication $(n=1027)$} \\
\hline Yes & 211 & $20.5 \pm 1.3$ \\
\hline No & 816 & $79.5 \pm 1.3$ \\
\hline \multicolumn{3}{|c|}{${ }^{a}$ Not all participants contributed data for all measures. } \\
\hline \multicolumn{3}{|c|}{$\begin{array}{l}\text { Scores range from } 0 \text { to } 8 \text {; a higher score indicates a higher degree } \\
\text { of hopelessness. }\end{array}$} \\
\hline
\end{tabular}

available. Model 1 is adjusted for age and baseline IMT score only; Model 2 is adjusted for all covariates found to be significantly related to change in IMT. A strong association was found between high baseline hopelessness and hostility and 4-year progression of IMT. Greater hostility was associated with greater 4-year change in mean and maximum IMT for both Model 1 and Model 2. Similarly, high hopelessness individuals were characterized by greater 4-year change for each IMT measure. Tables II and III include an assessment, per Everson et al. (1997a), of the relation between mean IMT scores by hopelessness category for those individuals with baseline IMT measures greater than the median.

\section{Analysis of Effect Modification}

SBP was dichotomized into low-moderate and high groups, since the low and moderate SBP tertiles had similar distributions for IMT, while the high SBP tertile presented a different distribution. Both models indicated significant effect modification by SBP of the association between hostility scores and 4-year change in mean IMT, maximum IMT and plaque height. Partial $F$ tests for effect modification of the hostility $\times$ SBP term were statistically significant for change in IMT measures. For mean IMT, $F=7.55(p<0.001)$; for maximum IMT, $F=$ $5.07(p=0.007)$; and for plaque height, $F=3.83$ $(p=0.02)$. The hostility $\times$ fibrinogen and hostility $\times$ WHR terms were not significant. Interactions with hopelessness were not significant.

For men in the lower tertiles of SBP, change in the three IMT measures in the moderate hostility group was similar to those of the low hostility group, while men in the high hostility group had the largest (albeit not statistically significant) gain in IMT measures (Fig. 1). Among men in the highest SBP tertile, the moderate hostility group had greater 4year IMT progression than men in the low hostility and the high hostility groups $(p=0.05)$. Figures $1(\mathrm{a})$ through 1(c) illustrate the different hostility-IMT relationships among the men in the highest SBP tertile compared to those in the lower two tertiles.

Table II. Mean 4-Year Change (95\% Confidence Interval) in Intima-Medial Thickness (IMT) for Affective Measures: Model $1^{a}$

\begin{tabular}{llll}
\hline & \multicolumn{1}{c}{ Mean IMT } & \multicolumn{1}{c}{ Maximum IMT } & \multicolumn{1}{c}{ Plaque height } \\
\hline Hostility & $n=923$ & $n=923$ & $n=921$ \\
Low & $0.095(0.079,0.110)$ & $0.238(0.216,0.260)$ & $0.253(0.233,0.272)$ \\
Moderate & $0.119(0.104,0.135)$ & $0.270(0.248,0.291)$ & $0.280(0.260,0.299)$ \\
High & $0.125^{*}(0.110,0.141)$ & $0.278^{*}(0.256,0.300)$ & $0.273(0.253,0.293)$ \\
Hopelessness & $n=992$ & $n=992$ & $n=990$ \\
Low/moderate & $0.111(0.102,0.121)$ & $0.260(0.246,0.273)$ & $0.268(0.256,0.280)$ \\
High & $0.136(0.106,0.166)$ & $0.309^{*}(0.267,0.352)$ & $0.297(0.259,0.336)$ \\
Hopelessness (baseline IMT $>$ median) & $n=508$ & $n=506$ & $n=508$ \\
Low/moderate & $0.093(0.077,0.110)$ & $0.239(0.215,0.262)$ & $0.209(0.190,0.229)$ \\
High & $0.132(0.084,0.181)$ & $0.326^{*}(0.254,0.398)$ & $0.241(0.178,0.303)$ \\
\hline
\end{tabular}

${ }^{a}$ Adjusted for age and baseline IMT.

${ }^{*} p<0.05$. 
Table III. Mean 4-Year Change (95\% Confidence Interval) in Intima-Medial Thickness (IMT) for Affective Measures: Model $2^{a}$

\begin{tabular}{llll}
\hline & \multicolumn{1}{c}{ Mean IMT } & \multicolumn{1}{c}{ Maximum IMT } & \multicolumn{1}{c}{ Plaque Height } \\
\hline Hostility & $n=873$ & $n=873$ & $n=871$ \\
Low & $0.095(0.079,0.111)$ & $0.240(0.218,0.263)$ & $0.258(0.238,0.279)$ \\
Moderate & $0.119^{*}(0.104,0.134)$ & $0.267^{*}(0.245,0.289)$ & $0.273(0.253,0.293)$ \\
High & $0.122^{*}(0.106,0.138)$ & $0.273^{*}(0.251,0.295)$ & $0.270(0.250,0.290)$ \\
Hopelessness & $n=940$ & $n=940$ & $n=938$ \\
low/moderate & $0.111(0.101,0.120)$ & $0.259(0.245,0.272)$ & $0.268(0.256,0.280)$ \\
high & $0.137(0.106,0.167)$ & $0.306^{*}(0.262,0.349)$ & $0.288^{*}(0.248,0.327)$ \\
Hopelessness (baseline IMT $>$ median) & $n=470$ & $n=471$ & $n=470$ \\
low/moderate & $0.093(0.076,0.109)$ & $0.236(0.213,0.260)$ & $0.203(0.184,0.223)$ \\
high & $0.134(0.083,0.186)$ & $0.321^{*}(0.246,0.397)$ & $0.226(0.162,0.290)$ \\
\hline
\end{tabular}

${ }^{a}$ Adjusted for baseline IMT, age, education, fibrinogen, WHR, SBP, smoking status, and treatment for hypertension. $* p<0.05$.

\section{Mediation by Biological Measures}

Analysis of change in beta coefficients implicated fibrinogen as the sole biological measure with
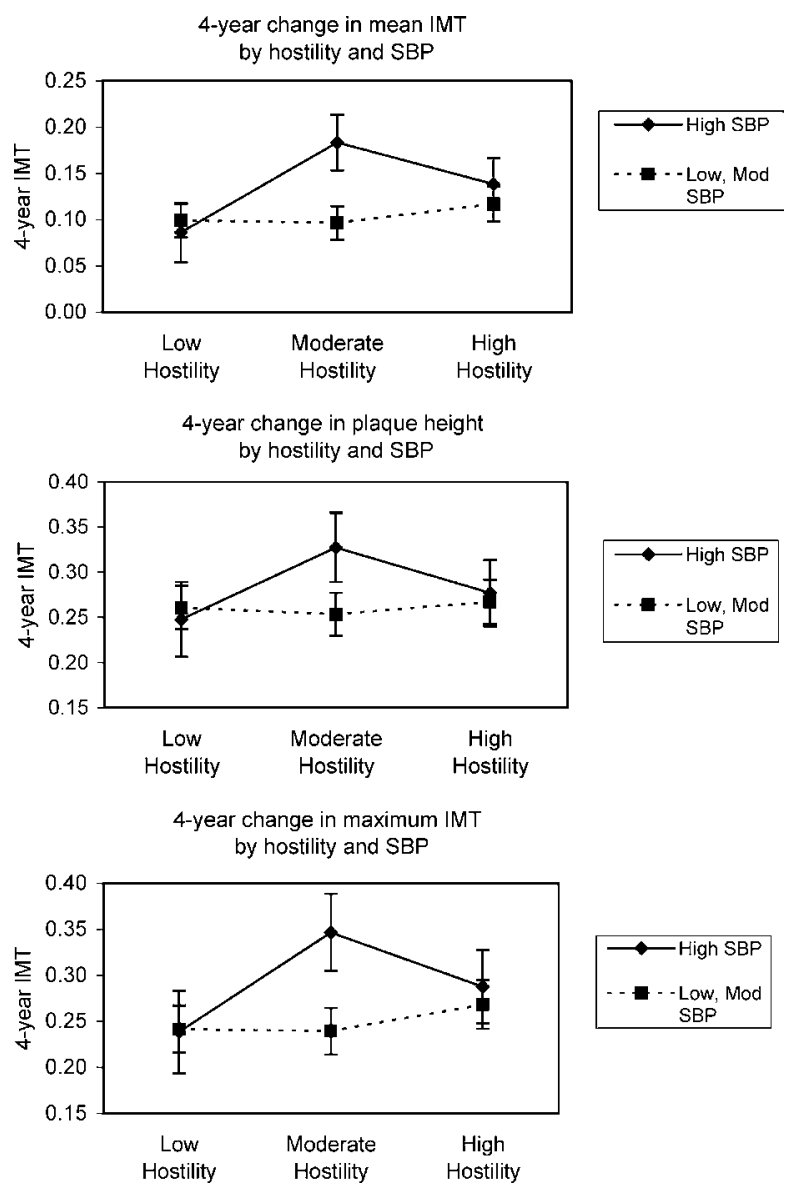

Fig. 1. Mean 4-year Change in IMT measurements, according to Baseline Hostility and Systolic Blood Pressure (SBP) (error bars correspond to $95 \%$ confidence interval). a role in mediating the hopelessness-IMT relationship. Hopelessness was a predictor of 4-year change in both mean and maximum IMT, for which the inclusion of fibrinogen in statistical models attenuated the extent to which the hopelessness measure was predictive of change in IMT. Table IV provides the percentage change in parameter estimates for hopelessness in models when fibrinogen was included. In Model 1 the percentage change in parameter estimates was 18.0, 13.1, and $8.5 \%$ for mean IMT, maximum IMT, and plaque height, respectively. None of these values approach the $30 \%$ change that might imply strong mediation; rather, percentage change in parameter estimates for mean IMT and maximum IMT suggest moderate mediation. The magnitude of parameter changes was reduced in Model 2 which controlled for all covariates. Hopelessness was not predictive of 4year change in plaque height with or without plasma fibrinogen in the model; thus percentage change in the beta coefficient for hopelessness-plaque height with fibrinogen in the model was not informative.

\section{DISCUSSION}

This analysis examined the possible modifying and mediating roles of three biological variablesplasma fibrinogen, resting SBP, and WHR-on the relationship between two negative affective states-hostility and hopelessness-and progression of carotid atherosclerosis in middle-aged men. Previous studies examining this cohort found that high baseline levels of hostility (Julkunen et al., 1994) and hopelessness (Everson et al., 1997a) were associated with increased PCA, and the present analysis confirmed these earlier reports. The novel and previously untested hypotheses examined by this analysis were that plasma fibrinogen, SBP, and WHR modify 
Table IV. Mediation by Plasma Fibrinogen of Relation Between Hopelessness and Change in IMT

\begin{tabular}{|c|c|c|c|c|}
\hline \multirow[b]{2}{*}{ 4-yr change } & \multicolumn{2}{|c|}{ Model $1^{a}$} & \multicolumn{2}{|c|}{ Model $2^{b}$} \\
\hline & Without fibrinogen & With fibrinogen & Without fibrinogen & With fibrinogen \\
\hline \multicolumn{5}{|l|}{ Mean IMT } \\
\hline Partial $F$-value & 3.57 & 2.40 & 2.82 & 2.13 \\
\hline Hopelessness $(p)$ & 0.06 & 0.12 & 0.09 & 0.14 \\
\hline $\operatorname{Beta}(\mathrm{SE})$ & $0.031(0.016)$ & $0.025(0.016)$ & $0.028(0.016)$ & $0.024(0.016)$ \\
\hline \% Change & & 18.0 & & 15.5 \\
\hline \multicolumn{5}{|l|}{ Maximum IMT } \\
\hline Partial $F$-value & 5.80 & 4.39 & 4.56 & 3.78 \\
\hline Hopelessness $(P)$ & 0.02 & 0.04 & 0.03 & 0.05 \\
\hline Beta (SE) & $0.056(0.023)$ & $0.048(0.023)$ & $0.049(0.023)$ & $0.045(0.023)$ \\
\hline \% Change & & 13.1 & & 10.1 \\
\hline \multicolumn{5}{|l|}{ Plaque height } \\
\hline Partial $F$-value & 1.82 & 1.51 & 0.89 & 0.83 \\
\hline Hopelessness $(P)$ & 0.18 & 0.22 & 0.34 & 0.36 \\
\hline Beta (SE) & $0.028(0.021)$ & $0.026(0.021)$ & $0.020(0.021)$ & $0.019(0.021)$ \\
\hline$\%$ Change & & 8.5 & & 4.2 \\
\hline
\end{tabular}

or mediate associations between hostility and hopelessness and PCA, such that accounting for the role of biological variables in associations between negative affect and PCA could further understanding of the underlying mechanisms. Strong effect modification by baseline SBP of the relation between hostility and PCA was observed; the impact of baseline hostility on 4-year progression of all IMT measures examined was significantly modified by SBP. Of the biological variables analyzed, plasma fibrinogen appeared to play a moderate role mediating the relationship between hopelessness and PCA.

\section{Effect Modification of Hostility-PCA by Systolic Blood Pressure}

A relationship between high hostility and elevated risk of CVD has been hypothesized to operate through the sympathetic nervous system or the hypothalamic-pituitary-adrenocortical axis. One of the biological measures known as influenced by hyperactivation of these two neuroendocrine pathways, SBP, was thus examined in this analysis for its potential role as an effect modifier. Our a priori hypothesis was that elevated SBP (as well as fibrinogen and WHR), possibly determined in part by the physiological responses of the organism to negative affective states, might operate to increase the effect of negative affective states on the 4-year progression of IMT. Our results indicate that SBP appears to be a strong effect modifier of the hostility-PCA relationship.
The strongest effect modification observed in this analysis concerned greater 4-year PCA among moderate hostility men with high SBP. Moderate hostility men experienced significantly greater PCA if in the high SBP group as opposed to the lowmoderate SBP group. Low and high hostility men, however, did not experience significantly different PCA based upon SBP. Thus the hypothesis of effect modification by SBP was partially confirmed.

Effect modification among the moderate hostility group was an unexpected finding for which various post hoc explanations can be posited. One such possibility is differential underlying propensities towards perceiving "challenge" situations, and corresponding differences in the frequency and magnitude of physiological responses. Men with elevated hostility scores have been shown by some, but not all, studies to experience an acute physiological response (e.g., rapid release of catecholamines, raised blood pressure and release of cortisol) to mentally stressful situations (Brunner, 1997; Carroll et al., 1997; Suls and Wan, 1993). Low-hostility individuals may perceive the balance of potentially stressful interpersonal situations as neither confrontational nor particularly challenge provoking, thus experiencing infrequent and/or minimal responses. Very cynical, highly hostile individuals, at the other extreme, may be inured to the occurrence of what they perceive as challenge-provoking situations according to their beliefs about other people's behavior, and thus also experience infrequent and/or minimal physiological responses in such situations. Moderate 
hostility individuals, however, may perceive and respond readily and frequently to a wide variety of interpersonal challenges. Elevated blood pressure in such individuals, then, may interact with the chronic hyperactivity of the SNS and HPA axes resulting from such repeated responses, thus providing an explanation for the increased PCA observed among this group. Additional studies are required to replicate the nature of effect modification as observed in this analysis.

\section{Mediation of Hopelessness-PCA by Plasma Fibrinogen Level}

Plasma fibrinogen seems to operate as a moderate-strength mediator of the hopelessnessPCA relation in this analysis. Fibrinogen is a marker of the systemic inflammation characterizing atherogenesis (Mosesson et al., 2001; Ross, 1999; Saadeddin et al., 2002). Its apparent mediation of the hopelessness-PCA association here is consistent with its role as an inflammatory marker. Injury to vascular endothelial cells due to hyperactivation of the HPA axis may result in higher plasma fibrinogen level. This reading of the mediating role of fibrinogen implied by this analysis does not ascribe a direct physiological role to fibrinogen in the pathway between hopelessness and PCA. Instead, an increase in fibrinogen may serve as a marker for cell damage, partially due to hopelessness-induced HPA hyperactivity, which occurs before vascular damage becomes measurable through change in IMT thickness.

An alternate interpretation of the mediating role of fibrinogen demonstrated by this analysis is that hopelessness-induced hyperactivation of the HPA axis results in an increase in plasma fibrinogen, which is then responsible for some of the progression of carotid atherosclerosis. The possibility that high hopelessness may result in elevated plasma fibrinogen level is consistent with the allostatic load model, wherein psychosocial stressors cause chronic activation of the HPA axis and may result in elevated risk of CVD (McEwen, 1998; McEwen and Seeman, 1999). Studies based on this model have found that low social class and high job stress, both risk factors for CVD, are related to high fibrinogen level (Markowe et al., 1985; Wilson et al., 1993). As fibrinogen plays a role in the early stages of plaque formation, an increase in plasma fibrinogen could result in greater progression of carotid atherosclerosis over time. This second interpretation of the me- diation analysis results places fibrinogen directly in the biological path linking hopelessness and carotid atherosclerosis. Unfortunately, the design of this exploratory analysis does not allow for distinguishing between these two interpretations of mediation. Studies that specifically examine physiological mechanisms linking negative psychosocial states and progression of atherosclerosis are needed to further describe the role that plasma fibrinogen and other biological measures play in such relationships. It may be that fibrinogen is both directly and indirectly involved in mediating the link between hopelessness and PCA. Specifically how and why hopelessness might be linked, via fibrinogen, to the inflammatory process also requires further study.

The present analysis was limited in its ability to determine a possible mediating role of plasma fibrinogen due to sample size limitations. Everson et al. reported that the association between hopelessness and PCA was much greater among individuals whose baseline IMT was above the median (Everson et al., 1997a). We were unable to examine the potential mediating role of fibrinogen among men with baseline IMT above the median separately from individuals with baseline IMT below the median, as cell sizes became too small for multivariate analysis when this further dichotomization was made. Fibrinogen may play a significantly greater role among individuals for whom baseline IMT is in the upper half of the distribution; however, detection of a possibly greater mediating role of plasma fibrinogen among these individuals was not practicable. Additionally, a 4-year follow-up period may not be adequate, as documenting mediation of the pathophysiologic effects operating through the HPA and sympathoadrenal pathways may necessitate a longer follow-up period for such effects to be observed.

\section{Analytic Design}

Criticism has been leveled at analyses such as this which seek to separate the total effect of an exposure (e.g., hostility or hopelessness) on a given outcome (e.g., PCA) into indirect and direct effects (Cole and Hernan, 2002; Poole and Kaufman, 2000; Robins and Greenland, 1992). The argument goes as follows: If there is confounding by an unmeasured variable of the relation between a mediator and an outcome, then conditioning on the exposureoutcome association according to the level of the mediator may lead to an inaccurate division of the total effect into "indirect" and "direct" components. 
Blakely (2002) has noted that examples used to make the case that adjusting for mediators leads to an inaccurate partitioning of a total effect have involved data either 1) simulated or hypothetical, or 2) taken from actual studies chosen for an extreme distribution of population characteristics (Blakely, 2002). Therefore, until new and realistic examples serve to indicate that the estimation of direct and indirect effects can lead to flawed conclusions in common scenarios, abandoning this method may well be premature. Instead, the collection of data on possible confounders, the inclusion of such data in analyses, and consideration of assumptions that go along with effect decomposition can serve as useful safeguards against inaccurate conclusions (Cole and Hernan, 2002). Effect decomposition was performed in this exploratory analysis with an awareness of the potential methodological problems involved with this technique. Recommended safeguards (Blakely, 2002; Cole and Hernan, 2002) have been employed towards assuring the valid estimation of the mediating effects of the variables examined. Attention has been given to potential confounding of the causal intermediate in the biological processes under study. Further epidemiologic and physiological studies examining the activity of plasma fibrinogen in the pathway between negative psychosocial states and CHD are needed to confirm the mediating role suggested by this analysis.

\section{CONCLUSION}

This exploratory analysis attempted to discern the roles of biological variables in pathophysiological pathways that may operate parallel to one another, may interact with each other, and may also be influenced by unknown confounders. The HPA axis and the sympathoadrenal system have each been implicated in the association between the negative affective states of depression and hostility and elevated risk for CVD. Such implication is quite different, however, from a precise explication of the roles that each pathway plays in the link between affective states and CVD. The extent to which each pathway influences psychosomatic associations, and the possible interaction of these two pathways, has yet to be elucidated. The present report supports a moderate role for fibrinogen as a mediator of the link between hopelessness and PCA. It also provides support for SBP as modifying the relation between hostility and PCA. As new studies specifically aimed at determin- ing the operation of the implicated pathways are undertaken, a shift from exploratory analyses to a more precise explication of roles of particular biological measures will allow for a clearer description of the connections between affective states such as depression and an elevated risk of CVD.

\section{ACKNOWLEDGMENTS}

We would like to thank Dr Riitta M. Salonen, $\mathrm{MD}, \mathrm{PhD}$, for supervising the ultrasonographic assessment of atherosclerosis and Kimmo Ronkainen, MPH, for data management. Dr Jukka Salonen was partially funded by a grant from the Academy of Finland.

\section{REFERENCES}

Alloy, L. B., Abramson, L. Y., Metalsky, G. I., and Hartlage, S. (1988). The hopelessness theory of depression: attributional aspects. Br. J. Clin. Psychol. 27(Pt. 1): 5-21.

Anda, R., Williamson, D., Jones, D., Macera, C., Eaker, E., Glassman, A., and Marks, J. (1993). Depressed affect, hopelessness, and the risk of ischemic heart disease in a cohort of U.S. adults. Epidemiology 4(4): 285-294.

Aromaa, A., Raitasalo, R., Reunanen, A., Impivaara, O., Heliovaara, M., Knekt, P., Lehtinen, V., Joukamaa, M., and Maatela, J. (1994). Depression and cardiovascular diseases. Acta Psychiatr. Scand. Suppl. 377: 77-82.

Barefoot, J. C., Larsen, S., von der Lieth, L., and Schroll, M. (1995). Hostility, incidence of acute myocardial infarction, and mortality in a sample of older Danish men and women. Am. J. Epidemiol. 142(5): 477-484.

Baron, R. M., and Kenny, D. A. (1986). The moderator-mediator variable distinction in social psychological research: Conceptual, strategic, and statistical considerations. J. Pers. Soc. Psychol. 51(6): 1173-1182.

Bjorntorp, P. (1997). Stress and cardiovascular disease. Acta Physiol. Scand. Suppl. 640: 144-148.

Blakely, T. (2002). Commentary: Estimating direct and indirect effects-fallible in theory, but in the real world? Int. J. Epidemiol. 31(1): 166-167.

Bollen, K. (1998). Path analysis. In Armitage, P., and Colton, T. (Eds.), Encyclopedia of Biostatistics, Vol. 4, Wiley, New York.

Brown, G. W., and Harris, T. (1978). Social Origins of Depression, Kluwer Academic Publishers, Tavistock, London.

Bruce, M. L., Leaf, P. J., Rozal, G. P., Florio, L., and Hoff, R. A. (1994). Psychiatric status and 9-year mortality data in the New Haven Epidemiologic Catchment Area Study. Am. J. Psychiatry 151(5): 716-721.

Bruhn, J. G., Paredes, A., Adsett, C. A., and Wolf, S. (1974). Psychological predictors of sudden death in myocardial infarction. J. Psychosom. Res. 18(3): 187-191.

Brunner, E. (1997). Stress and the biology of inequality. BMJ 314(7092): 1472-1476

Carroll, D., Davey, S. G., Sheffield, D., Shipley, M. J., and Marmot, M. G. (1997). The relationship between socioeconomic status, hostility, and blood pressure reactions to mental stress in men: Data from the Whitehall II study. Health Psychol. 16(2): 131-136.

Checkley, S. (1996). The neuroendocrinology of depression and chronic stress. Br. Med. Bull. 52(3): 597-617. 
Clauss, A. (1957). Gerinnungshphysiologische Schnellmethode zur Bestimmung des fibrinogens. (In German.) Acta Haematol. (Basel) 17: 237-246.

Cohen, S., and Rodriguez, M. S. (1995). Pathways linking affective disturbances and physical disorders. Health Psychol. 14: 374-380.

Cole, S. R., and Hernan, M. A. (2002). Fallibility in estimating direct effects. Int. J. Epidemiol. 31(1): 163-165.

Cook, W. W., and Medley, D. M. (1954). Proposed hostility and pharisaic-virtue scales for the MMPI. J. Appl. Psychol. 38: 414-418.

Crouse, J. R., III. (2001). Predictive value of carotid 2-dimensional ultrasound. Am. J. Cardiol. 88(2-A): 27E-30E.

Crouse, J. R., Goldbourt, U., Evans, G., Pinsky, J., Sharrett, A. R., Sorlie, P., Riley, W., and Heiss, G. (1996). Risk factors and segment-specific carotid arterial enlargement in the Atherosclerosis Risk in Communities (ARIC) cohort. Stroke 27(1): 69-75.

Engel, G. L. (1968). A life setting conducive to illness. The givingup-given-up complex. Bull. Menninger. Clin. 32(6): 355365.

Everson, S. A., Goldberg, D. E., Kaplan, G. A., Cohen, R. D., Pukkala, E., Tuomilehto, J., and Salonen, J. T. (1996). Hopelessness and risk of mortality and incidence of myocardial infarction and cancer. Psychosom. Med. 58(2): 113121.

Everson, S. A., Kaplan, G. A., Goldberg, D. E., Salonen, R., and Salonen, J. T. (1997a). Hopelessness and 4-year progression of carotid atherosclerosis. The Kuopio Ischemic Heart Disease Risk Factor Study. Arterioscler. Thromb. Vasc. Biol. 17(8): 1490-1495.

Everson, S. A., Kauhanen, J., Kaplan, G. A., Goldberg, D. E., Julkunen, J., Tuomilehto, J., and Salonen, J. T. (1997b). Hostility and increased risk of mortality and acute myocardial infarction: The mediating role of behavioral risk factors. Am. J. Epidemiol. 146(2): 142-152.

Ferland, M., Despres, J. P., Tremblay, A., Pinault, S., Nadeau, A., Moorjani, S., Lupien, P. J., Theriault, G., and Bouchard, C. (1989). Assessment of adipose tissue distribution by computed axial tomography in obese women: Association with body density and anthropometric measurements. $B r . J$. Nutr. 61(2): 139-148.

Glassman, A. H., Helzer, J. E., Covey, L. S., Cottler, L. B., Stetner, F., Tipp, J. E., and Johnson, J. (1990). Smoking, smoking cessation, and major depression. JAMA 264(12): 1546-1549.

Glassman, A. H., and Shapiro, P. A. (1998). Depression and the course of coronary artery disease. Am. J. Psychiatry 155(1): $4-11$.

Greene, S. M. (1989). The relationship between depression and hopelessness. Implications for current theories of depression. Br. J. Psychiatry 154: 650-659.

Greenglass, E. R., and Julkunen, J. (1991). Cook-Medley hostility, anger, and the Type A behavior pattern in Finland. Psychol. Rep. 68(3, Pt. 2): 1059-1066.

Hearn, M. D., Murray, D. M., and Luepker, R. V. (1989). Hostility, coronary heart disease, and total mortality: A 33-year followup study of university students. J. Behav. Med. 12(2): 105121.

Hoyle, R. H., and Kenny, D. A. (1999). Sample size, reliability, and test of statistical mediation. In Hoyle, R. H. (Ed.), Statistical Strategies for Small Sample Research, Sage, New York.

Iribarren, C., Sidney, S., Bild, D. E., Liu, K., Markovitz, J. H., Roseman, J. M., and Matthews, K. (2000). Association of hostility with coronary artery calcification in young adults: The CARDIA study. Coronary Artery Risk Development in Young Adults. JAMA 283(19): 2546-2551.

Julkunen, J., Salonen, R., Kaplan, G. A., Chesney, M. A., and Salonen, J. T. (1994). Hostility and the progression of carotid atherosclerosis. Psychosom. Med. 56(6): 519-525.
Kaplan, G. A., and Keil, J. E. (1993). Socioeconomic factors and cardiovascular disease: A review of the literature. Circulation 88(4, Pt. 1): 1973-1998.

Kelly, S., Hertzman, C., and Daniels, M. (1997). Searching for the biological pathways between stress and health. Annu. Rev. Public Health 18: 437-462.

Kenny, D. A., Kashy, D. A., and Bolger, N. (1998). Data analysis in social psychology. In Lindzey, G. (Ed.), The Handbook of Social Psychology, Vol. 1, McGraw-Hill, New York.

Keys, A. (1980). Seven Countries: A Multivariate Analysis of Death and Coronary Heart Disease, Harvard University Press, Cambridge, MA.

Kleinbaum, D. G., Kupper, L. K., Muller, K. E., and Nizam, A. (1998). Applied Regression Analysis and Other Multivariable Methods, 3rd ed., Brooks/Cole Publishing Company, Boston.

Kop, W. J. (1999). Chronic and acute psychological risk factors for clinical manifestations of coronary artery disease. Psychosom. Med. 61(4): 476-487.

Kop, W. J., Gottdiener, J. S., Tangen, C. M., Fried, L. P., McBurnie, M. A., Walston, J., Newman, A., Hirsch, C., and Tracy, R. P. (2002). Inflammation and coagulation factors in persons $>65$ years of age with symptoms of depression but without evidence of myocardial ischemia. Am. J. Cardiol. 89(4): 419-424.

Laghrissi-Thode, F., Wagner, W. R., Pollock, B. G., Johnson, P. C., and Finkel, M. S. (1997). Elevated platelet factor 4 and beta-thromboglobulin plasma levels in depressed patients with ischemic heart disease. Biol. Psychiatry 42(4): 290-295.

Lynch, J., Krause, N., Kaplan, G. A., Salonen, R., and Salonen, J. T. (1997). Workplace demands, economic reward, and progression of carotid atherosclerosis. Circulation 96(1): 302-307.

Lynch, J. W., Everson, S. A., Kaplan, G. A., Salonen, R., and Salonen, J. T. (1998). Does low socioeconomic status potentiate the effects of heightened cardiovascular responses to stress on the progression of carotid atherosclerosis? Am. J. Public Health 88(3): 389-394.

Lynch, J. W., Kaplan, G. A., Cohen, R. D., Tuomilehto, J., and Salonen, J. T. (1996). Do cardiovascular risk factors explain the relation between socioeconomic status, risk of all-cause mortality, cardiovascular mortality, and acute myocardial infarction? Am. J. Epidemiol. 144(10): 934-942.

Markowe, H. L., Marmot, M. G., Shipley, M. J., Bulpitt, C. J., Meade, T. W., Stirling, Y., Vickers, M. V., and Semmence, A. (1985). Fibrinogen: A possible link between social class and coronary heart disease. BMJ (Clin. Res. Ed.) 291(6505): 13121314.

Maruta, T., Hamburgen, M. E., Jennings, C. A., Offord, K. P., Colligan, R. C., Frye, R. L., and Malinchoc, M. (1993). Keeping hostility in perspective: Coronary heart disease and the Hostility Scale on the Minnesota Multiphasic Personality Inventory. Mayo Clin. Proc. 68(2): 109-114.

Matthews, K. A., Owens, J. F., Kuller, L. H., Sutton-Tyrrell, K., and Jansen-McWilliams, L. (1998). Are hostility and anxiety associated with carotid atherosclerosis in healthy postmenopausal women? Psychosom. Med. 60(5): 633-638.

Mayo-Smith, W., Hayes, C. W., Biller, B. M., Klibanski, A., Rosenthal, H., and Rosenthal, D. I. (1989). Body fat distribution measured with CT: Correlations in healthy subjects, patients with anorexia nervosa, and patients with Cushing syndrome. Radiology 170(2): 515-518.

McEwen, B. S. (1998). Protective and damaging effects of stress mediators. N. Engl. J. Med. 338(3): 171-179.

McEwen, B. S., and Seeman, T. (1999). Protective and damaging effects of mediators of stress. Elaborating and testing the concepts of allostasis and allostatic load. Ann. N.Y. Acad. Sci. 896: 30-47.

McEwen, B. S., and Stellar, E. (1993). Stress and the individual. Mechanisms leading to disease. Arch. Int. Med. 153(18): 2093-2101. 
Miller, G. E., Stetler, C. A., Carney, R. M., Freedland, K. E., and Banks, W. A. (2002). Clinical depression and inflammatory risk markers for coronary heart disease. Am. J. Cardiol. 90(12): 1279-1283.

Mosesson, M. W., Siebenlist, K. R., and Meh, D. A. (2001). The structure and biological features of fibrinogen and fibrin. Ann. N. Y. Acad. Sci. 936: 11-30.

Musselman, D. L., Evans, D. L., and Nemeroff, C. B. (1998). The relationship of depression to cardiovascular disease: Epidemiology, biology, and treatment. Arch. Gen. Psychiatry 55(7): 580-592.

Musselman, D. L., Tomer, A., Manatunga, A. K., Knight, B. T., Porter, M. R., Kasey, S., Marzec, U., Harker, L. A., and Nemeroff, C. B. (1996). Exaggerated platelet reactivity in major depression. Am. J. Psychiatry 153(10): 1313-1317.

Niedhammer, I., Goldberg, M., Leclerc, A., David, S., Bugel, I., and Landre, M. F. (1998). Psychosocial work environment and cardiovascular risk factors in an occupational cohort in France. J. Epidemiol. Commun. Health 52(2): 93-100.

Poole, C., and Kaufman, J. S. (2000). What does standard adjustment for downstream mediators tell us about social effect pathways? Am. J. Epidemiol. 151: S52.

Pope, M. K., and Smith, T. W. (1991). Cortisol excretion in high and low cynically hostile men. Psychosom. Med. 53(4): 386-392.

Pratt, L. A., Ford, D. E., Crum, R. M., Armenian, H. K., Gallo, J. J., and Eaton, W. W. (1996). Depression, psychotropic medication, and risk of myocardial infarction. Prospective data from the Baltimore ECA follow-up. Circulation 94(12): 3123-3129.

Ritcher, C. (1957). On the phenomenon of sudden death in animals and man. Psychosom. Med. 19: 190-198.

Robins, J. M., and Greenland, S. (1992). Identifiability and exchangeability for direct and indirect effects. Epidemiology 3(2): 143-155.

Ross, R. (1999). Atherosclerosis-An inflammatory disease. $N$. Engl. J. Med. 340(2): 115-126.

Rozanski, A., Blumenthal, J. A., and Kaplan, J. (1999). Impact of psychological factors on the pathogenesis of cardiovascular disease and implications for therapy. Circulation 99(16): 2192-2217.

Rugulies, R. (2002). Depression as a predictor for coronary heart disease. a review and meta-analysis. Am. J. Prev. Med. 23(1): $51-61$.
Saadeddin, S. M., Habbab, M. A., and Ferns, G. A. (2002) Markers of inflammation and coronary artery disease. Med. Sci. Monit. 8(1): RA5-RA12.

Salonen, J. T. (1988). Is there a continuing need for longitudinal epidemiologic research? The Kuopio Ischaemic Heart Disease Risk Factor Study. Ann. Clin. Res. 20(1-2): 46-50.

Salonen, J. T., Korpela, H., Salonen, R., and Nyyssonen, K. (1993). Precision and reproducibility of ultrasonographic measurement of progression of common carotid artery atherosclerosis. Lancet 341(8853): 1158-1159.

Salonen, R., and Salonen, J. T. (1993). Intima-media changes in a population study: KIHD. In Boccalon, H. (Ed.), Vascular Medicine, Elsevier Science, Amsterdam.

Selzer, R. H., Hodis, H. N., Kwong-Fu, H., Mack, W. J., Lee, P. L. Liu, C. R., and Liu, C. H. (1994). Evaluation of computerized edge tracking for quantifying intima-media thickness of the common carotid artery from B-mode ultrasound images. Atherosclerosis 111(1): 1-11.

Suarez, E. C., and Blumenthal, J. A. (1991). Ambulatory blood pressure responses during daily life in high and low hostile patients with a recent myocardial infarction. J. Cardiopulm. Rehabil. 11: 169-175.

Suarez, E. C., Kuhn, C. M., Schanberg, S. M., Williams, R. B., Jr., and Zimmermann, E. A. (1998). Neuroendocrine, cardiovascular, and emotional responses of hostile men: The role of interpersonal challenge. Psychosom. Med. 60(1): 78-88.

Suls, J., and Wan, C. K. (1993). The relationship between trait hostility and cardiovascular reactivity: A quantitative review and analysis. Psychophysiology 30(6): 615-626.

Tsigos, C., and Chrousos, G. P. (2002). Hypothalamic-pituitaryadrenal axis, neuroendocrine factors and stress. J. Psychosom. Res. 53(4): 865-871.

Twisk, J. W., Kemper, H. C., Van Mechelen, W., and Post, G. B. (2001). Clustering of risk factors for coronary heart disease. The longitudinal relationship with lifestyle. Ann. Epidemiol. 11(3): 157-165.

Wilson, T. W., Kaplan, G. A., Kauhanen, J., Cohen, R. D., Wu, M., Salonen, R., and Salonen, J. T. (1993). Association between plasma fibrinogen concentration and five socioeconomic indices in the Kuopio Ischemic Heart Disease Risk Factor Study. Am. J. Epidemiol. 137(3): 292-300.

Ziegelstein, R. C., Bush, D. E., and Fauerbach, J. A. (1998) Depression, adherence behavior, and coronary disease outcomes. Arch. Int. Med. 158(7): 808-809. 